\title{
Cameras for Stereo Panoramic Imaging *
}

\author{
Shmuel Peleg Yael Pritch Moshe Ben-Ezra \\ School of Computer Science and Engineering \\ The Hebrew University of Jerusalem \\ 91904 Jerusalem, ISRAEL
}

\begin{abstract}
A panorama for visual stereo consists of a pair of panoramic images, where one panorama is for the left eye, and another panorama is for the right eye. A panoramic stereo pair provides a stereo sensation up to a full $360 \mathrm{de}$ grees. A stereo panorama cannot be photographed by two omnidirectional cameras from two viewpoints. It is normally constructed by mosaicing together images from a rotating stereo pair, or from a single moving camera. Capturing stereo panoramic images by a rotating camera makes it impossible to capture dynamic scenes at video rates, and limits stereo panoramic imaging to stationary scenes.

This paper presents two possibilities for capturing stereo panoramic images using optics, without any moving parts. A special mirror is introduced such that viewing the scene through this mirror creates the same rays as those used with the rotating cameras. Such a mirror enables the capture of stereo panoramic movies with a regular video camera. A lens for stereo panorama is also introduced. The designs of the mirror and of the lens are based on curves whose caustic is a circle.
\end{abstract}

\section{Introduction}

The ultimate immersive visual environment should provide three elements: (i) Stereo vision, where each eye gets a different image appropriate to its location in space; (ii) complete 360 degrees view, allowing the viewer to look in any desired direction; (iii) allow free movement.

Stereo Panoramas $[6,5,10,14]$ use a new scene to image projection that enables simultaneously both (i) stereo and (ii) a complete panoramic view. No depth information or correspondences are necessary. Viewers of stereo panoramas have the ability to freely view, in stereo, all di-

${ }^{*}$ This research was partially funded by European ESPRIT project 26247-VIGOR, as well as by Israel Science Foundation Grant 612/97. Contact E-Mail: \{peleg,moshe,yaelpri\}@cs.huji.ac.il rections.

Since the scene to image projection necessary for stereo panoramic imaging can not be done with a regular camera, stereo panoramic images were generated by mosaicing images taken with rotating cameras $[6,5,10,14]$. As it is necessary to rotate a video camera a full circle in order to obtain a single stereo panoramic images, it was impossible to generate video-rate stereo panoramic movies.

In this paper we present two possible camera systems, without any moving parts, that can capture stereo panoramic movies in video rate. One system uses special mirrors, and the other system uses special lenses. With such cameras it will be possible to make stereo panoramic movies of real events: sports, travel, etc.

Short introductions are given in this section to panoramic imaging, stereo imaging, multiple viewpoint projection$\mathrm{s}$, and caustic curves. Sec. 2 discusses the multiple viewpoint projection that can be used to create stereo panoramas. Sec. 3 describes the method to create stereo panoramas using rotating cameras. Sec. 4 describes the generation of stereo panoramas using static mirrors. Sec. 5 describes the generation of full 360 degrees stereo panoramas using static lens.

\subsection{Panoramic Images}

A panoramic image is a wide field of view image, up to a full view of 360 degrees. Panoramas can be created on an extended planar image surface, on a cylinder, or on a sphere. Traditional panoramic images have a single viewpoint, also called the "center of projection" $[8,3,15]$. Panoramic images can be captured by panoramic cameras, by using special mirrors $[9,7]$, or by mosaicing a sequence of images from a rotating camera $[15,11]$.

\subsection{Visual Stereo}

A stereo pair consists of two images of a scene from two different viewpoints. The disparity, which is the angular difference in viewing directions of each scene point between the two images, is interpreted by the brain as depth. Fig. 1 describes a conventional stereo setting. The disparity 

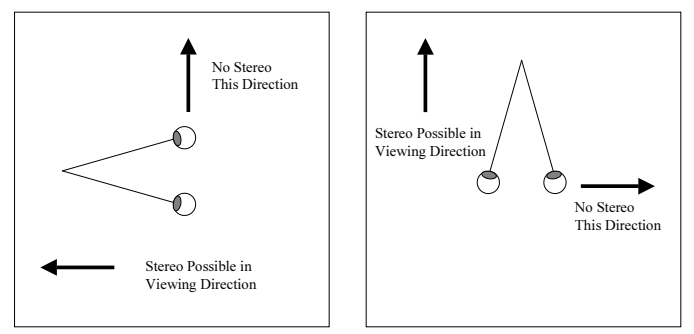

Figure 1. No arrangement of two singleviewpoint images can give stereo in all viewing directions. For upward viewing the two cameras should be separated horizontally, and for sideways viewing the two cameras should be separated vertically.

is a function of the point's depth and the distance between the eyes (baseline). Maximum disparity change, and hence maximum depth separation, is along the line in the scene whose points have equal distances from both eyes ("principal viewing direction"). No stereo depth separation exists for points along the extended baseline.

People can perceive depth from stereo images if the viewpoints of the two cameras generate horizontal disparity in a specific range. Stereo has been obtained in panoramic images by having two viewpoints, one above the other [4]. However, since the disparity in this case is vertical, it can only be used for depth calculation, and not for viewing by humans having eyes which are separated horizontally.

\subsection{Caustic curves}

Definition 1 The envelope of a set of curves is a curve $C$ such that $C$ is tangent to every member of the set.

Definition 2 A caustic is the envelope of rays emanating from a point source and reflected (or refracted) by a given curve.

A caustic curve caused by reflection is called a catacaustic, and a caustic curve caused by refraction is called a diacaustic [17]. In Fig. 8 the catacaustic curve given the mirror and the optical center is a circle. In Fig. 10 and Fig. 11, the diacaustic curve given the lens and the optical center is a circle.

\section{Multiple Viewpoint Projections}

Regular images are created by perspective projection$s$ : scene points are projected onto the image surface along projection lines passing through a single point, called the "optical center" or the "viewpoint". Multiple viewpoint projections use different viewpoints for different viewing

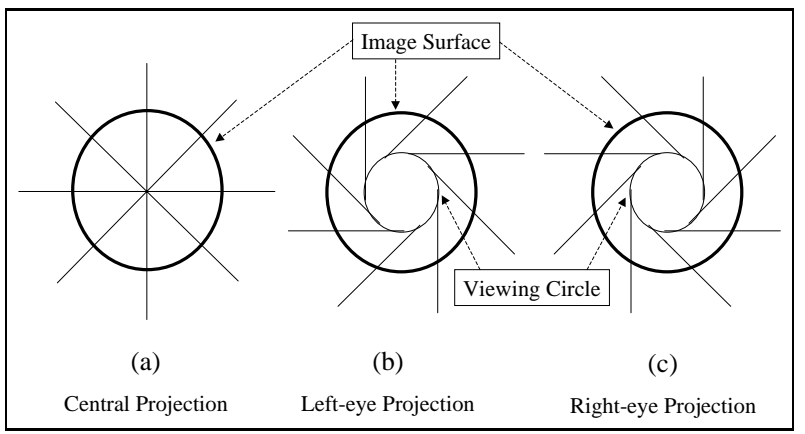

Figure 2. Circular projections. The projection from the scene to the image surface is done along the rays tangent to the viewing circle. (a) Projection lines perpendicular to the circular imaging surface create the traditional single-viewpoint panoramic image. (b-c) Families of projection lines tangent to the inner viewing circle form the multipleviewpoint circular projections.

direction, and were used mostly for special mosaicing applications. Effects that can be created with multiple viewpoint projections and mosaicing are discussed in $[16,12]$.

Stereo panoramic imaging uses a special type of multiple viewpoint projections, circular projections, where both the left-eye image and the right-eye image share the same cylindrical image surface. To enable stereo perception, the left viewpoint and the right viewpoint are located on an inner circle (the "viewing circle") inside the cylindrical image surface, as shown in Fig. 2. The viewing direction is on a line tangent to the viewing circle. The left-eye projection uses the rays on the tangent line in the clockwise direction of the circle, as in Fig. 2.b. The right-eye projection uses the rays in the counter clockwise direction as in Fig. 2.c. Every point on the viewing circle, therefore, defines both a viewpoint and a viewing direction of its own.

The applicability of circular projections to panoramic stereo is shown in Fig. 3. From this figure it is clear that the two viewpoints associated with all viewing directions, using the "left-eye" projection and the "right-eye" projection, are in optimal relative positions for stereo viewing for all directions. The vergence is also identical for all viewing directions [13], unlike regular stereo that has a preferred viewing direction.

\section{Stereo Panoramas with Rotating Cameras}

Representing all stereoscopic views with only two panoramic images presents a contradiction, as described in Fig. 1. When two ordinary panoramic images are captured from two different viewpoints, the disparity and the stereo 


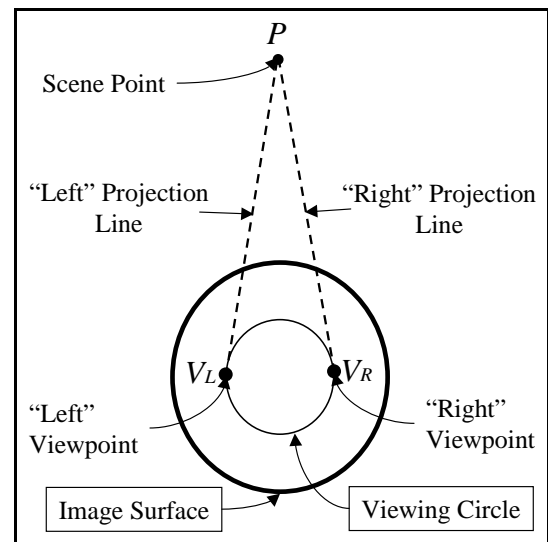

Figure 3. Viewing a scene point with "lefteye" and "right-eye" projections. The two viewpoints for these two projections are always in optimal positions for stereo viewing.

perception will degrade as the viewing direction becomes closer to the baseline until no stereo will be apparent.

Generation of Image-based stereo panoramas by rotating a stereo head having two cameras was proposed in $[5,14]$. A stereo head with two cameras is rotated, and two panoramic mosaics are created from the two different cameras. A Single rotating camera can also be sufficient under some conditions $[10,6,14]$. In the case of a single moving camera different sides of the same image are used to mosaic the two images for the different eyes. This can even be done in real-time [10] (See Fig. 4).

\section{Stereo Panoramas with a Spiral Mirror}

Regular cameras are designed to have a single viewpoint ("optical center"), following the perspective projection. In this section we show how to create images having circular projections using a regular camera and a spiral shaped mirror.

The shape of the spiral mirror can be determined for a given optical center of the camera $o$, and a desired viewing circle $V$. The tangent to the mirror at every point has equal angles to the optical center and to the tangent to the circle (See Fig. 5). Each ray passing through the optical center will be reflected by this mirror to be tangent to the viewing circle. This is true also in reverse: all rays tangent to the circle will be reflected to pass through the optical center. The mirror is therefore a curve whose catacaustic is a circle.

The conditions at a surface patch of the spiral shaped mirror are shown in Fig. 6. The optical center is at the center of the viewing circle of radius $R$, and the mirror is defined by its distance $r(\theta)$ from the optical center. A

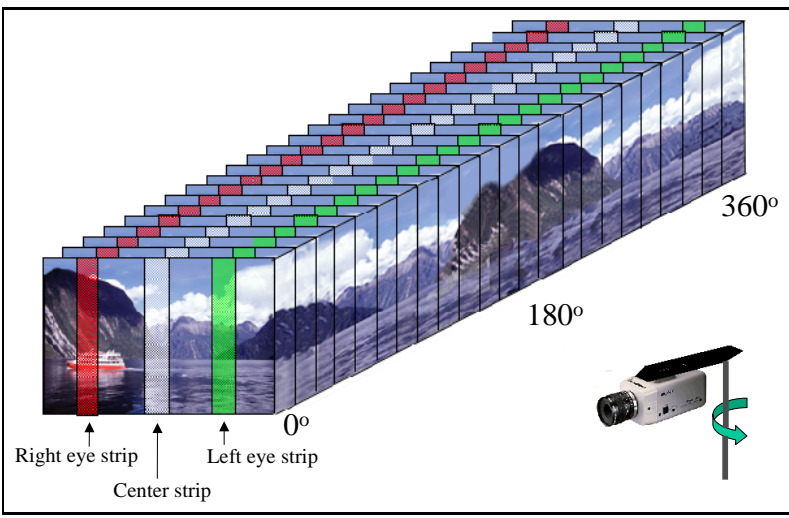

Figure 4. Stereo Panoramas can be created using images captured with a regular camera rotating about an axis behind it. Pasting together strips taken from each image approximates the panoramic image cylinder. When the strips are taken from the center of the images an ordinary panorama is obtained. When the strips are taken from the left side of each image, the viewing direction is tilted counter clockwise from the image surface, obtaining the right-eye panorama. When the strips are taken from the right side of each image, the left-eye panorama is obtained.

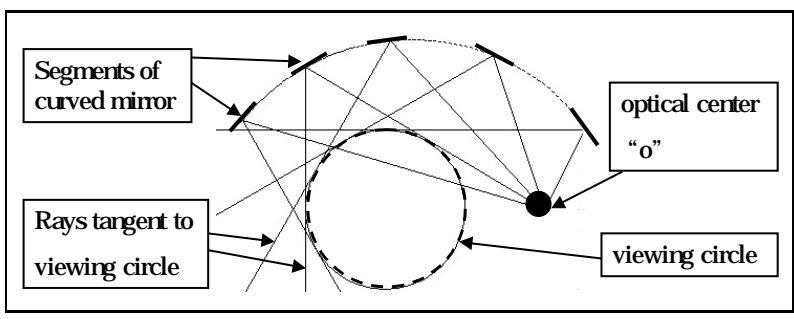

Figure 5. The spiral mirror: All rays passing through the optical center $o$ will be reflected by the mirror to be tangent to the viewing circle $V$. This implies that rays tangent to the viewing circle will be reflected to pass through the optical canter. 
ray passing through the optical center hits the mirror at an angle $\alpha$ to the normal, and is reflected to be tangent to the viewing circle.

Let the radius of the viewing circle be $R$, and denote by $\bar{r}(\theta)$ the vector from the optical center and the mirror at direction $\theta$ (measured from the $x$-axis). The distance between the camera center and the mirror at direction $\theta$ will therefore be $r=r(\theta)=\|\bar{r}\|$. The ray conditions can be written as:

$$
\begin{aligned}
& R=\|\bar{r}\| \sin (2 \alpha)=\|\bar{r}\| 2 \sin (\alpha) \cos (\alpha) \\
& \sin (\alpha)=\frac{|N \times \bar{r}|}{\|\bar{r}\| \cdot\|N\|} \\
& \cos (\alpha)=\frac{(N, r)}{\|\bar{r}\| \cdot\|N\|}
\end{aligned}
$$

using those conditions we can derive the following differential equation, where $\rho=\rho(\theta)$ is defined to be $\frac{r(\theta)}{R}$.

$$
2 \rho^{2} \frac{\partial \rho}{\partial \theta}=\left(\frac{\partial \rho}{\partial \theta}\right)^{2}+\rho^{2}
$$

This second degree equation in $\frac{\partial \rho}{\partial \theta}$ has two possible solutions:

$$
\frac{\partial \rho}{\partial \theta}=\left\{\begin{array}{c}
\rho^{2}+\rho \sqrt{\rho^{2}-1} \\
\rho^{2}-\rho \sqrt{\rho^{2}-1}
\end{array}\right\} .
$$

The curve is obtained by integration on $\theta$. The solution which fits our case is:

$$
\theta=\rho+\sqrt{\rho^{2}-1}+\arctan \left(\frac{1}{\sqrt{\rho^{2}-1}}\right)
$$

With the constraint that $\rho>1$.

The spiral mirror can also be represented by a parametric equation. Given the position of the camera $\left(p_{1}, p_{2}\right)$ and the radius $R$ of a viewing circle centered around the origin, points $(x(t), y(t))$ on the mirror can be represented as a function of a parameter $t$ :

$$
\begin{aligned}
& x=\frac{\sin (t)\left(R^{2}+p_{1}{ }^{2}-R^{2} t^{2}+p_{2}{ }^{2}\right)-2 p_{2} R-2 R^{2} t \cos (t)}{2\left(-p_{2} \cos (t)-R t+\sin (t) p_{1}\right)} \\
& y=\frac{-\cos (t)\left(R^{2}+p_{1}{ }^{2}-R^{2} t^{2}+p_{2}{ }^{2}\right)+2 p_{1} R-2 R^{2} t \sin (t)}{2\left(-p_{2} \cos (t)-R t+\sin (t) p_{1}\right)}
\end{aligned}
$$

When the camera is positioned at the origin, e.g. in the center of the viewing circle, the equations above simplify to:

$$
\begin{aligned}
& x=\frac{R\left(-\sin (t)+2 t \cos (t)+t^{2} \sin (t)\right)}{2 t} \\
& y=\frac{R\left(\cos (t)+2 t \sin (t)-t^{2} \cos (t)\right)}{2 t}
\end{aligned}
$$

A curve satisfying these conditions has a spiral shape, and Fig 7 shows such a curve extended for three cycles.

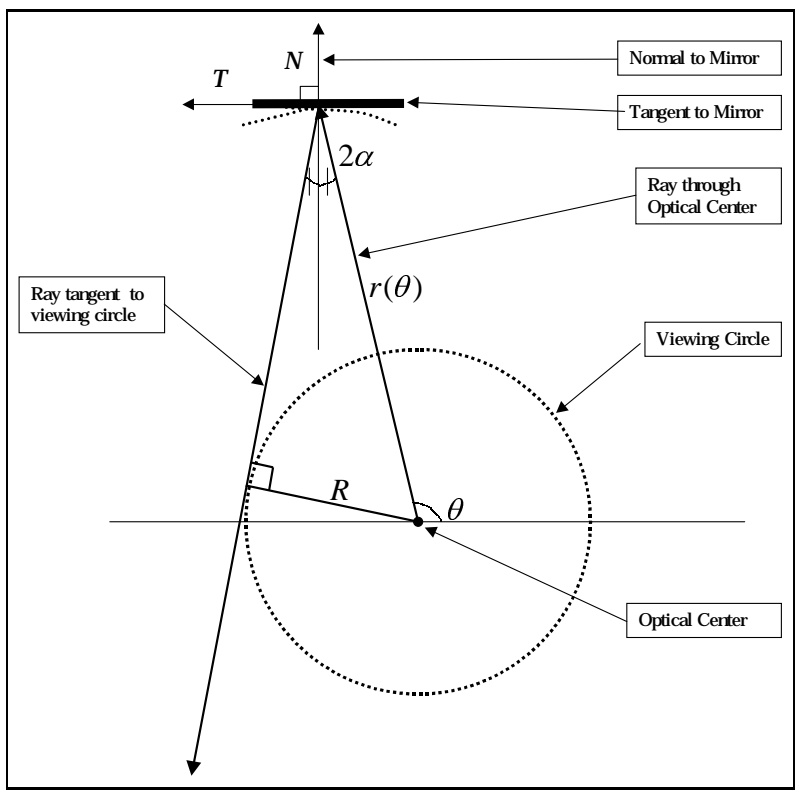

Figure 6. Differential conditions at a mirror patch: The optical center is at the center of the viewing circle of radius $R$, and the mirror is defined by its distance $r(\theta)$ from the optical center. A ray passing through the optical center hits the mirror at an angle $\alpha$ to the normal, and is reflected to be tangent to the viewing circle.

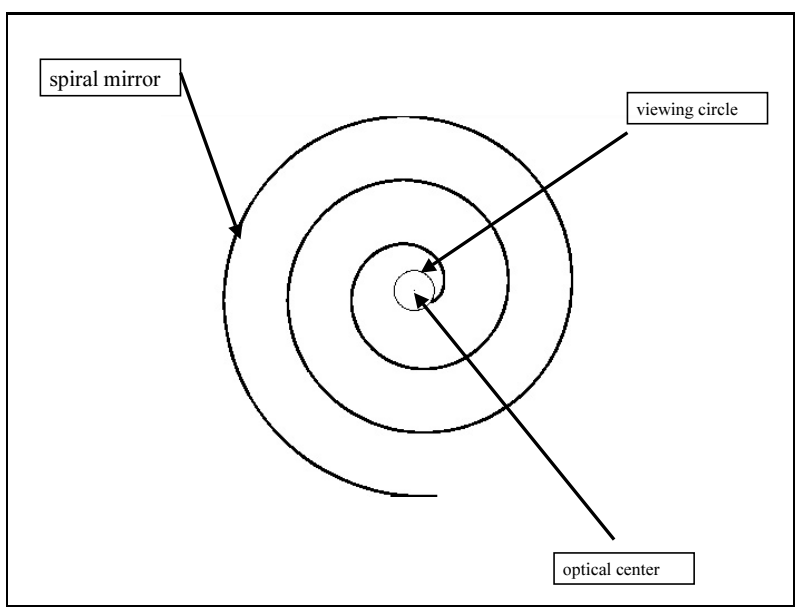

Figure 7. A spiral shaped mirror extended for three full cycles. The catacaustic curve of this spiral is the small inner circle. 


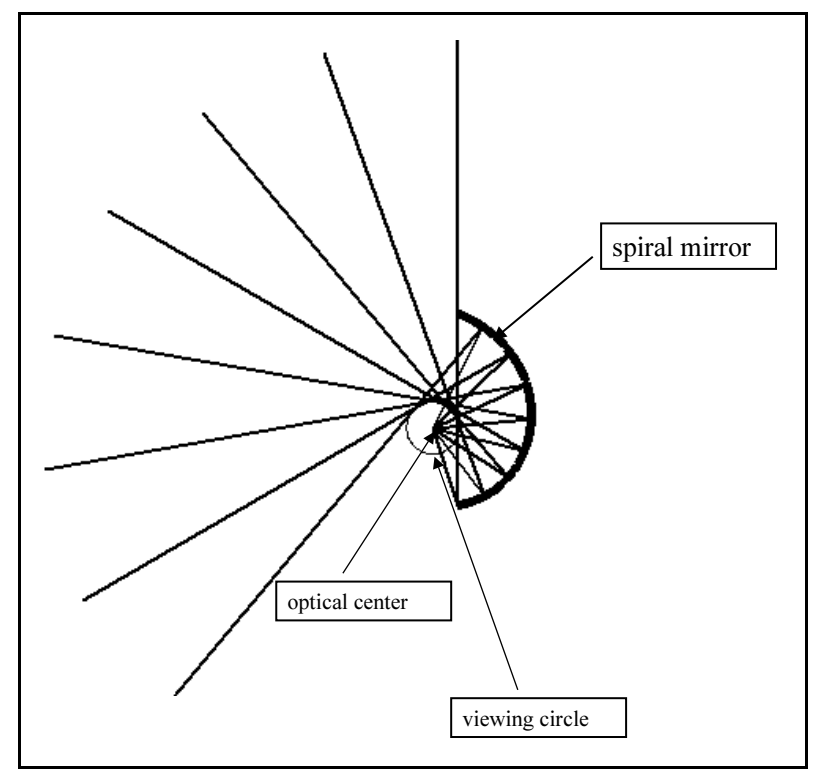

Figure 8. A spiral mirror where the optical center is at the center of the viewing circle.

To avoid self occlusion, a practical mirror will use only segments of this curve.

A spiral shaped mirror where the optical center is located at the center of the viewing circle is shown in Fig. 8.

The configuration where the optical center is at the center of the viewing circle is also convenient for imaging together the left image and the right image. Such a symmetric configuration is shown in Fig. 9. This configuration has a mirror symmetry, and each mirror covers approximately 132 degrees without self occlusions. An Omni Camera $[9,7]$ can be placed at the center of the viewing circle to capture both the right image and the left image. Since this setup captures up to approximately 132 degrees, three such cameras are necessary to cover a full 360 degrees.

\section{Stereo Panoramas with a Spiral Lens}

Circular projections can also be obtained with a lens whose diacaustic is a circle: the lens refracts the rays getting out of the optical center to be tangent to the viewing circle, as shown in Fig. 10. A lens can cover up to 360 degrees without self occlusion depending on the configuration. The spiral of the lens is different from the spiral of the mirror. We have not yet computed an explicit expression for this curve, and it is generated using numerical approximations.

It is possible to simplify the configuration and use multiple identical segments of a spiral lens, each capturing a small angular sector. Fig. 11 presents a configuration of fifteen lenses, each covering 24 degrees. The concept of

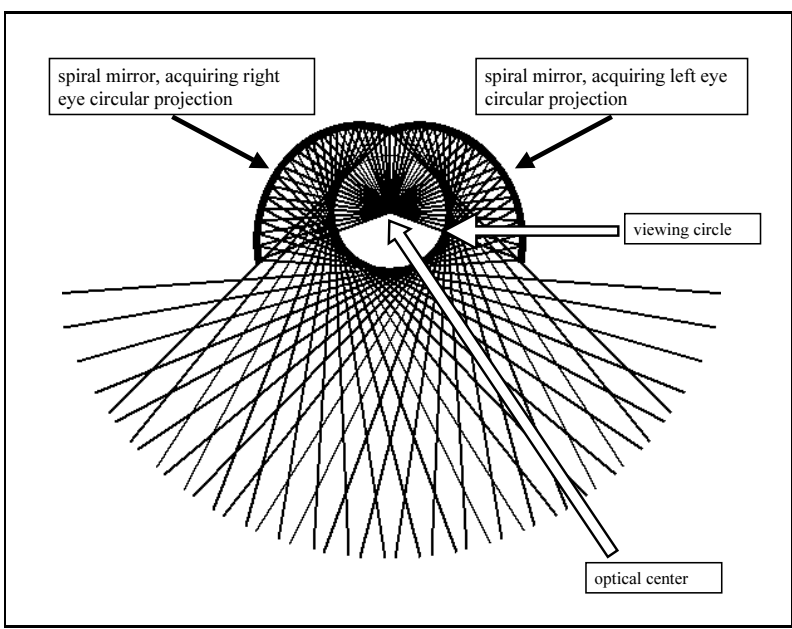

Figure 9. Two spiral shaped mirrors sharing the same optical center and the viewing circle. One mirror for the left-circular-projection and one for the right-circular-projection.

switching from one big lens to multiple smaller lenses that produce the same optical function was first used in the Fresnel lens. In practice, a Fresnel-like lens can be constructed for this purpose having thousands of segments. A convenient way to view the entire panorama is by placing a panoramic omnidirectional camera $[9,7]$ at the center of the lens system as shown in Fig. 12.

A camera setup for creating two 360 degrees panoramas simultaneously (one for each eye) is presented in Fig. 13. This system consists of two multiple-lens systems as described in Fig. 11. A parabolic beam splitter and a parabolic mirror are used. The beam splitter splits each of the rays to two separate identical rays. The rays which are not reflected by the beam splitter enter the lower lens system. The rays which are reflected by the beam splitter are reflected again by the mirror into the upper lens system. One lens system will produce a panoramic images using a left viewing circle, and the other lens system will produce a panoramic image using a right viewing circle.

The requirement that the cylindrical optical elemen$t$ (e.g. as in Fig. 11) just bends the rays in the horizontal direction is accurate for rays that are in the same plane of the viewing circle. But this is only an approximation for rays that come from different vertical directions as shown in Fig. 14. Let us examine the rays for viewpoint $R$. Ray $A$ is in the horizontal plane that includes the viewing circle $V$. It is deflected by the Fresnel lens into ray $a$, and passes through the center $O$ of the viewing circle, the location of the optical center of the panoramic camera. Ray $B$, which also passes through viewpoint $R$, but from a higher ele- 


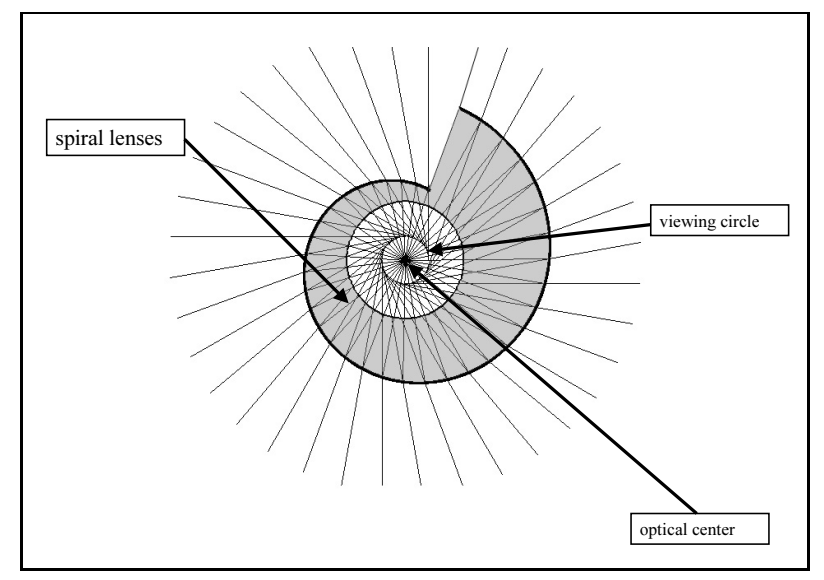

Figure 10. A spiral shaped lens. The diacaustic of the lens' outer curve is a circle (the viewing circle). Capturing the panorama can be done by an omnidirectional camera at the center of the viewing circle.

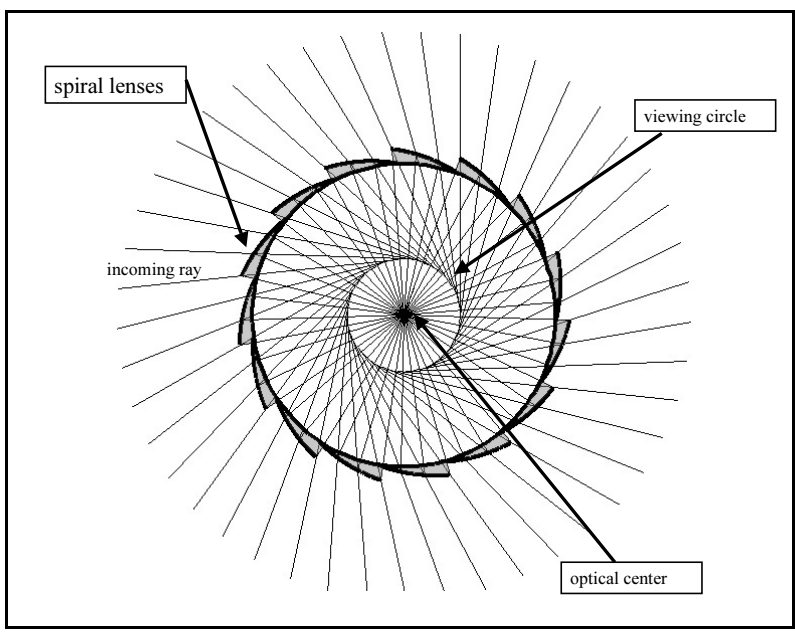

Figure 11. A collection of identical short spiral lens positioned on a circle. A Fresnel-like lens can be built with thousands of lens segments. Capturing the panorama can be done by an Omni Camera at the center of the viewing circle.

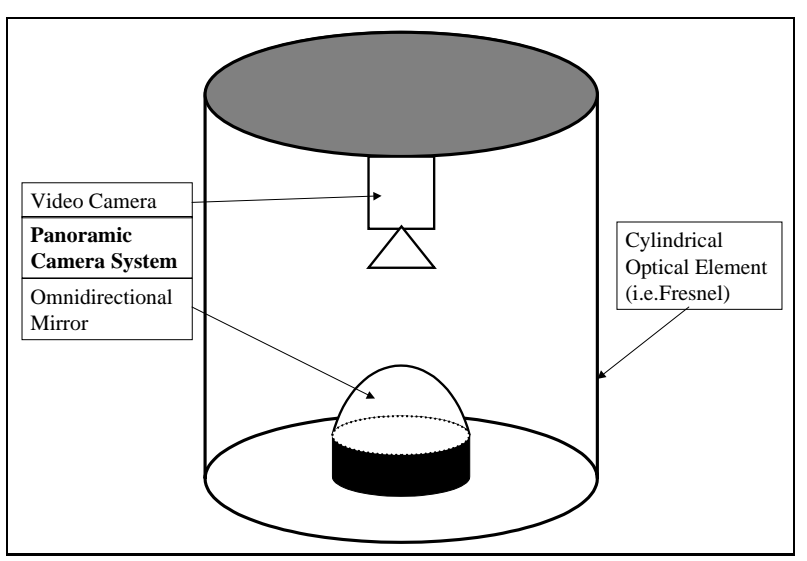

Figure 12. An omnidirectional camera at the center of the viewing circle enables the creation of a full 360 degrees left-image or a right-image.

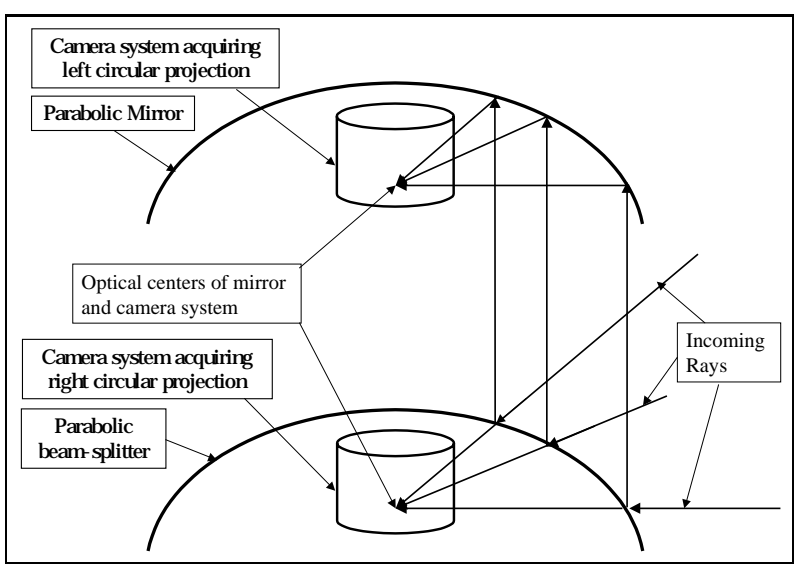

Figure 13. A setup for simultaneous capture of both left and right panoramas. A beam splitter is used to split the rays between two lens system. The horizontal rays enter into the bottom lens system for the right eye. The upward rays are reflected by a mirror into the upper lens system for the left eye. 


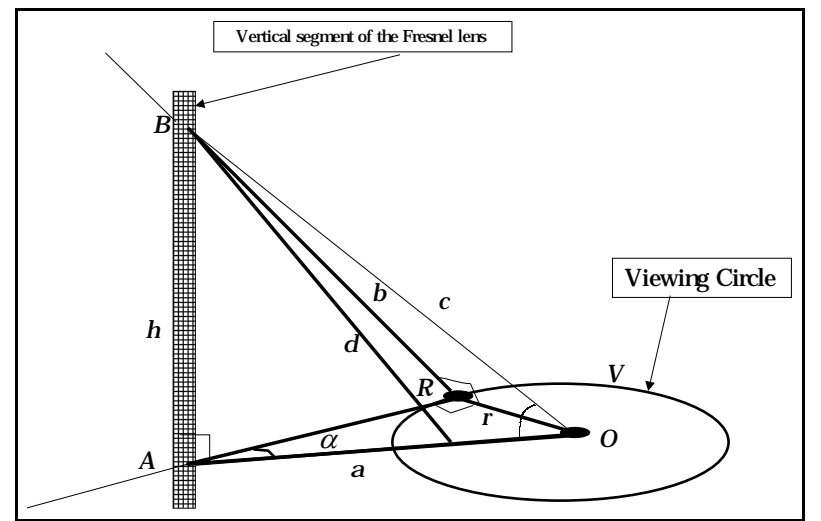

Figure 14. Vertical deflection of rays is necessary in order to assure that every viewing direction will have a single viewpoint on the viewing circle.

vation, is also deflected by the same horizontal angle, but will not reach $O$. Instead, Ray $B$ is deflected into ray $d$, which intersects the horizontal plane closer to the Fresnel lens than $O$. In order that ray $B$ will be deflected into Ray $c$, that intersects $O$, the Fresnel lens should deflect it also in the vertical direction. Each elevation should have a different vertical deflection. A possible arrangement is that the cylindrical Fresnel lens has vertical elements on one side that take care of the horizontal deflection (which is constant), and on the other side it has horizontal elements that take care of the horizontal deflection (which is different for every elevation).

\section{Concluding Remarks}

Two systems, having no moving parts, were presented for capturing stereo panoramic video. One system is based on spiral mirrors, and the second system is based on spiral lenses. While not constructed yet at the time of writing this paper, these systems represent the only known possibilities to capture real-time movies having the stereo panoramic features.

\section{Acknowledgements}

The authors wish to thank Tanya Matskewich (Hebrew University) and Eisso Atzema (University of Maine) for their help in deriving the expressions defining the spiral mirror.

\section{Bibliography}

[1] IEEE Conference on Computer Vision and Pattern Recognition, San Juan, Puerto Rico, June 1997.

[2] Seventh International Conference on Computer Vision, Kerkyra, Greece, September 1999. IEEE-CS.
[3] S. Chen. Quicktime VR - an image-based approach to virtual environment navigation. In SIGGRAPH'95, pages 2938, Los Angeles, California, August 1995. ACM.

[4] J. Gluckman, S. Nayar, and K. Thoresz. Real-time omnidirectional and panoramic stereo. In DARPA IUW'98, pages 299-303, Monterey, California, November 1998. Morgan Kaufmann.

[5] H.-C. Huang and Y.-P. Hung. Panoramic stereo imaging system with automatic disparity warping and seaming. Graphical Models and Image Processing, 60(3):196-208, May 1998.

[6] H. Ishiguro, M. Yamamoto, and S. Tsuji. Omni-directional stereo. IEEE Trans. on Pattern Analysis and Machine Intelligence, 1992.

[7] T. Kawanishi, K. Yamazawa, H. Iwasa, H. Takemura, and N. Yokoya. Generation of high-resolution stereo panoramic images by omnidirectional sensor using hexagonal pyramidal mirrors. In 14th International Conference on Pattern Recognition, pages 485-489, Brisbane, Australia, August 1998. IEEE-CS.

[8] S. Mann and R. Picard. Virtual bellows: Constructing high quality stills from video. In First IEEE International Conference on Image Processing, volume I, pages 363-367, Austin, Texas, November 1994.

[9] S. Nayar. Catadioptric omnidirectional cameras. In IEEE Conference on Computer Vision and Pattern Recognition [1], pages 482-488.

[10] S. Peleg and M. Ben-Ezra. Stereo panorama with a single camera. In IEEE Conference on Computer Vision and Pattern Recognition, pages 395-401, Ft. Collins, Colorado, June 1999.

[11] S. Peleg and J. Herman. Panoramic mosaics by manifold projection. In IEEE Conference on Computer Vision and Pattern Recognition [1], pages 338-343.

[12] P. Rademacher and G. Bishop. Multiple-center-ofprojection images. In SIGGRAPH'98, pages 199-206, Orlando, Florida, July 1998. ACM.

[13] H. Shum, A. Kalai, and S. Seitz. Omnivergent stereo. In Seventh International Conference on Computer Vision [2], pages 22-29.

[14] H. Shum and R. Szeliski. Stereo reconstruction from multiperspective panoramas. In Seventh International Conference on Computer Vision [2], pages 14-21.

[15] R. Szeliski. Video mosaics for virtual environments. IEEE Computer Graphics and Applications, 16(2):22-30, 1996.

[16] D. Wood, A. Finkelstein, J. Hughes, C. Thayer, and D. Salesin. Multiperspective panoramas for cel animation. In SIGGRAPH'97, pages 243-250, Los Angeles, California, August 1997. ACM.

[17] R. C. Yates. A Handbook on Curves and Their Properties, rev. ed. National Council of Teachers of Mathematics, 1952, reprinted 1974. 